\title{
326.
}

\section{THEOREMS RELATING TO THE CANONIC ROOTS OF A BINARY QUANTIC OF AN ODD ORDER.}

[From the Philosophical Magazine, vol. xxv. (1863), pp. 206-208.]

I CALL to mind Professor Sylvester's theory of the canonical form of a binary quantic of an odd order; viz., the quantic of the order $2 n+1$ may be expressed as a sum of a number $n+1$ of $(2 n+1)$ th powers, the roots of which, or say the canonic roots of the quantic, are to constant multipliers près the factors of a certain covariant derivative of the order $(n+1)$, called the Canonizant. If, to fix the ideas, we take a quintic function, then we may write

$$
\left(a, b, c, d, e, f \gamma(x, y)^{5}=A(l x+m y)^{5}+A^{\prime}\left(l^{\prime} x+m^{\prime} y\right)^{5}+A^{\prime \prime}\left(l^{\prime \prime} x+m^{\prime \prime} y\right)^{5}\right.
$$

(it would be allowable to put the coefficients $A$ each equal to unity; but there is a convenience in retaining them, and in considering that a canonic root $l x+m y$ is only given as regards the ratio $l: m$, the coefficients $l, m$ remaining indeterminate); and then the canonic roots $(l x+m y)$, \&c. are the factors of the Canonizant

$$
\left|\begin{array}{cccr}
y^{3}, & -y^{2} x, & y x^{2}, & -x^{3} \\
a, & b, & c, & d \\
b, & c, & d, & e \\
c, & d, & e, & f
\end{array}\right|
$$

It is to be observed that this reduction of the quantic to its canonical form, i.e. to a sum of a number $n+1$ of $(2 n+1)$ th powers, is a unique one, and that the quantic cannot be in any other manner a sum of a number $n+1$ of $(2 n+1)$ th powers. 
Professor Sylvester communicated to me, under a slightly less general form, and has permitted me to publish the following theorems:

1. If the second emanant $\left(X \partial_{x}+Y \partial_{y}\right)^{2} U$ has in common with the quantic $U$ a single canonic root, then all the canonic roots of the emanant are canonic roots of the quantic; and, moreover, if the remaining canonic root of the quantic be $r x+s y$, then $(X, Y)$, the facients of emanation, are $=(s,-r)$, or, what is the same thing, they are given by the equation

$$
\text { canont. } U(X, Y \text { in place of } x, y)=0 \text {. }
$$

In fact, considering, as before, the quintic $U=\left(a, b, c, d, e, f \gamma(x, y)^{5}\right.$, we have

$$
U=A(l x+m y)^{5}+A^{\prime}\left(l^{\prime} x+m^{\prime} y\right)^{5}+A^{\prime \prime}\left(l^{\prime \prime} x+m^{\prime \prime} y\right)^{5},
$$

and thence

$$
\left(X \partial_{x}+Y \partial_{y}\right)^{2} U=B(l x+m y)^{3}+B^{\prime}\left(l^{\prime} x+m^{\prime} y\right)^{3}+B^{\prime \prime}\left(l^{\prime \prime} x+m^{\prime \prime} y\right)^{3},
$$

if for shortness

$$
B=6.5(l X+m Y)^{2} A, \& c .
$$

Suppose $\left(X \partial_{x}+Y \partial_{y}\right)^{2} U$ has in common with $U$ the canonic root $l x+m y$, then

$$
\left(X \partial_{x}+Y \partial_{y}\right)^{2} U=C(l x+m y)^{3}+C^{\prime}(p x+q y)^{3},
$$

and thence

$$
B^{\prime}\left(l^{\prime} x+m^{\prime} y\right)^{3}+B^{\prime \prime}\left(l^{\prime \prime} x+m^{\prime \prime} y\right)^{3}=(C-B)(l x+m y)^{3}+C^{\prime}(p x+q y)^{3},
$$

which must be an identity; for otherwise we should have the same cubic function expressed in two different canonical forms. And we may write

$$
B^{\prime}=C^{\prime}, \quad l^{\prime} x+m^{\prime} y=p x+q y, \quad B^{\prime \prime}=0, \quad C=B,
$$

and then we have

$$
\left(X \partial_{x}+Y \partial_{y}\right)^{2} U=B(l x+m y)^{3}+B^{\prime}\left(l^{\prime} x+m^{\prime} y\right)^{3}
$$

so that all the canonic roots of the emanant are canonic roots of the quantic. Moreover, the condition $B^{\prime \prime}=0$ gives $l^{\prime \prime} X+m^{\prime \prime} Y=0$, that is, $X: Y=m^{\prime \prime}:-l^{\prime \prime}$, or writing $r x+s y$ instead of $l^{\prime \prime} x+m^{\prime \prime} y, X: Y=s:-r$; and the system is

$$
\begin{aligned}
U & =A(l x+m y)^{5}+A^{\prime}\left(l^{\prime} x+m^{\prime} y\right)^{5}+A(r x+s y)^{5}, \\
\left(s \partial_{x}-r \partial_{y}\right)^{2} U & =B(l x+m y)^{3}+B^{\prime}\left(l^{\prime} x+m^{\prime} y\right)^{3},
\end{aligned}
$$

which proves the theorem.

2. The two functions, canont. $U$, canont. $\left(X \partial_{x}+Y \partial_{y}\right)^{2} U$, have for their resultant \{canont. $U(X, Y$ in place of $x, y)\}^{2 n}$, if $2 n+1$ be the order of $U$.

In fact, in order that the equations

$$
\text { canont. } U=0 \text {, canont. }\left(X \partial_{x}+Y \partial_{y}\right)^{2} U=0 \text {, }
$$


may coexist, their resultant must vanish; and conversely, when the resultant vanishes, the equations will have a common root. Now if the equation canont. $\left(X \partial_{x}+Y \partial_{y}\right)^{2} U=0$ has a common root with the equation canont. $U=0$, all its roots are roots of canont. $U=0$; and, moreover, if $r x+s y=0$ be the remaining root of canont. $U=0$, then $X: Y=s:-r$, that is, we have

$$
\text { canont. } U(X, Y \text { in place of } x, y)=0 \text {; }
$$

or the resultant in question can only vanish if the last-mentioned equation is satisfied. It follows that the resultant must be a power of the nilfactum of the equation; and observing that canont. $U$ is of the form $(a, \ldots)^{n+1}(x, y)^{n+1}$, i.e. that it is of the degree $n+1$ as well in regard to the coefficients as in regard to the variables $(x, y)$, it is easy to see that the resultant is of the degree $2 n(n+1)$ as well in regard to the coefficients as in regard to $(X, Y)$; that is, we have $2 n$ as the index of the power in question.

3. In particular, if $Y=0$, the theorem is that the resultant of the functions canont. $U$, canont. $\partial_{x}{ }^{2} U$ is equal to the $2 n$th power of the first coefficient of canont. $U$.

Thus for $n=1$, that is, for the cubic function $\left(a, b, c, d \gamma(x, y)^{3}\right.$, we have

$$
\begin{aligned}
& \text { canont. } U=\left|\begin{array}{ccc}
y^{2}, & -x y, & x^{2} \\
a, & b, & c \\
b, & c, & d
\end{array}\right|=\left(a c-b^{2}, a d-b c, b d-c^{2} \gamma(x, y)^{2}\right. \text {, } \\
& \text { canont. } \partial_{x}{ }^{2} U=\left|\begin{array}{rr}
y, & -x \\
a, & b
\end{array}\right|=a x+b y
\end{aligned}
$$

and the resultant of the two functions is

$$
\begin{aligned}
& =\left(a c-b^{2}, \quad a d-b c, \quad b d-c^{2} \gamma b,-a\right)^{2} \\
& =-\left(a c-b^{2}\right)^{2},
\end{aligned}
$$

which verifies the theorem.

The theorems were, in fact, given to me in relation to the quantic $U$ and the second differential coefficient $\partial_{x}{ }^{2} U$; but the introduction instead thereof of the second emanant $\left(X \partial_{x}+Y \partial_{y}\right)^{2} U$ presented no difficulty.

2, Stone Buildings, W.C., February 16, 1863.

C. V. 